Case Report

\title{
DAS181 Treatment of Severe Parainfluenza Virus 3 Pneumonia in Allogeneic Hematopoietic Stem Cell Transplant Recipients Requiring Mechanical Ventilation
}

\author{
B. Dhakal, ${ }^{1}$ A. D'Souza, ${ }^{1}$ M. Pasquini, ${ }^{1}$ W. Saber, ${ }^{1}$ T. S. Fenske, ${ }^{1}$ R. B. Moss, ${ }^{2}$ \\ W. R. Drobyski, ${ }^{1}$ P. Hari, ${ }^{1}$ and M. Z. Abidi ${ }^{3}$ \\ ${ }^{1}$ Division of Hematology Oncology, Department of Medicine, Medical College of Wisconsin, Milwaukee, WI 53226, USA \\ ${ }^{2}$ Ansun BioPharma, San Diego, CA 92121, USA \\ ${ }^{3}$ Division of Infectious Disease, Department of Medicine, Medical College of Wisconsin, Milwaukee, WI 53226, USA
}

Correspondence should be addressed to M. Z. Abidi; mabidi@outlook.com

Received 27 October 2015; Accepted 3 January 2016

Academic Editor: Ting Fan Leung

Copyright $\odot 2016$ B. Dhakal et al. This is an open access article distributed under the Creative Commons Attribution License, which permits unrestricted use, distribution, and reproduction in any medium, provided the original work is properly cited.

Parainfluenza virus (PIV) may cause life-threatening pneumonia in allogeneic hematopoietic stem cell transplant (HSCT) recipients. Currently, there are no proven effective therapies. We report the use of inhaled DAS181, a novel sialidase fusion protein, for treatment of PIV type 3 pneumonia in two allogeneic hematopoietic SCT recipients with respiratory failure.

\section{Introduction}

In allogeneic hematopoietic stem cell transplant (HSCT) recipients, parainfluenza (PIV) virus can cause varying spectrum of respiratory illness, ranging from self-limited upper respiratory tract infections (URTI) to severe, life-threatening lower respiratory tract infections (LRTI) $[1,2]$. In HSCT recipients and patients with hematologic malignancies, progression from URTI to LRTI is estimated to occur in 13\% to $43 \%$ of cases, whereas mortality rates from PIV LRTI are reported between $12 \%$ and 50\% [3]. Clinical manifestations may vary in severity based upon the type of transplant and immune suppression [4]. The mainstay of PIV treatment in HSCT recipients remains to be largely supportive care along with reduction of immune suppression, as currently there is a lack of antiviral agents or vaccines with proven efficacy [5]. DAS181 is a novel investigational sialidase fusion protein, which removes sialic acid-containing receptors from the surface of respiratory epithelial cells, and thus prevents PIV and influenza virus from binding to these cells [6]. DAS181 has been shown to be safe in phase I and II clinical trials for treatment of influenza [7] and also has also been shown to have in vitro and in vivo activity against PIV $[8,9]$. In recent years, there have been a very limited number of HSCT and lung transplant recipients with PIV LRTI treated with DAS181 with improvement in symptoms, viral loads, and oxygen requirements [10-14]. We describe use of DAS181 in two critically ill allogeneic HSCT recipients who presented with PIV type 3 (PIV3) LRTI.

\section{Methods}

Patients were diagnosed with PIV3 infection by viral nucleic acid amplification test (NAAT) done on nasopharyngeal swab and bronchoalveolar fluid lavage (BAL) samples. In each case, DAS181 (Ansun BioPharma, Inc., San Diego, California) was obtained through an emergency investigational new drug (EIND) application and was approved by the US Food and Drug Administration and the Medical College of Wisconsin Institutional Review Board. Informed consent was obtained from the patients' families. DAS181 was administered as a nebulized solution for 5-10 days. The appropriate dose was then administered over 10 minutes, based on manufacturer recommendations. Daily assessment was carried out for 
clinical and laboratory parameters and for any adverse events. The following laboratory tests were monitored while patients received treatment: complete blood count with differential, electrolytes, alkaline phosphatase, alanine aminotransferase, and aspartate aminotransferase. After completion of therapy complete blood counts and liver function tests were monitored on a weekly basis after completion of therapy.

Case 1. The patient was a 74-year-old female with myelodysplastic syndrome (MDS) who was treated with hypomethylating agents and then underwent a 7/8 HLA-mismatched related peripheral blood allogeneic SCT in April 2015 using reduced-intensity conditioning with melphalan, fludarabine, and $200 \mathrm{cGY}$ total body irradiation. She was on mycophenolate mofetil and tacrolimus for graft versus host disease (GVHD) prophylaxis. After transplant, she remained pancytopenic secondary to chemotherapy. On transplant day (TxD) +18 , the patient developed a new productive cough, fevers and was noted to be more tachypneic. She was found to be positive for PIV3 by viral NAAT on nasopharyngeal swab. Intravenous immune globulins (IVIG) and corticosteroid therapy were administered; however her respiratory status continued to worsen, requiring transfer to the intensive care unit for intubation on $\mathrm{TxD}+24$. Bronchoscopy with bronchoalveolar lavage (BAL) was performed and the NAAT for PIV3 was positive on the BAL fluid. DAS181 was obtained by EIND and was administered via Aeroneb nebulizer starting on $\mathrm{TxD}+25$. At onset of therapy, decision was made to limit treatment course of DAS181 for five days. The only appreciable side effect of DAS181 was an increase in alkaline phosphatase (ALK), which peaked at 288 (35104 units/Liter) at day 4 of therapy. Baseline ALK prior to start of therapy was 177 units/Liter. At the end of the 5-day course of DAS181, while she remained intubated, her oxygen requirements had improved. Her fraction of inspired oxygen (FiO2) requirements decreased to $40 \%$, positive end expiratory pressure (PEEP) 5 with oxygen saturation of $100 \%$. She subsequently displayed signs of sepsis, which was attributed to intravascular catheter infection with Staphylococcus epidermidis and was treated with IV linezolid. Despite receipt of growth factor she failed to have hematopoeitic recovery. Her fevers persisted with increased pressor requirements and she developed multiorgan failure. The decision was made by her family to withdraw care. She expired on day $\mathrm{TxD}+32$.

Case 2. A 35-year-old Caucasian woman presented with cough and shortness of breath at day $\mathrm{TxD}+324$ after myeloablative allogeneic matched unrelated donor peripheral blood SCT for acute myeloid leukemia in second complete remission. After transplant, she developed acute GVHD involving the skin and gut and subsequently chronic GVHD involving the skin and oral cavity as well as upper gastrointestinal tract in the form of eosinophilic esophagitis. Her ongoing immunosuppression regimen included corticosteroids, mycophenolate mofetil, and extracorporeal photopheresis. Computerized tomography (CT) scan of the chest showed new diffuse nodular infiltrates with multifocal consolidation bilaterally. Urine pneumococcal antigen was positive and she was started on intravenous (IV) antibiotics.
A viral NAAT done on a nasopharyngeal swab was positive for PIV3, and the patient received oral ribavirin for 7 days. On hospital day 4, she was discharged home on oral antibiotics and ribavirin. She was readmitted a week later, with worsening cough, dyspnea, and increased oxygen requirements and was empirically started on IV broad-spectrum antibiotics and underwent bronchoscopy with BAL. The BAL fluid showed PIV3 by NAAT, evidence of cytomegalovirus viral DNA, and 1,000 colony forming units/mL of Pseudomonas aeruginosa. Although blood CMV NAAT remained negative, she was started on IV ganciclovir and IVIG for possible CMV pneumonitis. She continued to worsen and required mechanical ventilation. On hospital day 6, DAS181 use was obtained and administered via an Aeroneb nebulizer. She received 10 days of therapy with DAS181 and we observed a decrease in her oxygen requirements during the course of therapy. Only side effect was an asymptomatic increase in ALK levels, which peaked at 261 (35-104 units/Liter) by day 6 of therapy. Prior to start of therapy, ALK was 88 units/Liter. This resolved spontaneously after completion of therapy. Her ICU stay was complicated by the development of Pseudomonas aeruginosa ventilator associated pneumonia on hospital day 21, which was successfully treated. A marked clinical and radiologic improvement was seen and she was discharged from the ICU and eventually extubated after a tracheostomy and a total of thirty days of mechanical ventilation.

\section{Discussion}

PIV infections cause substantial morbidity and mortality in SCT recipients and have no effective treatment $[5,15]$. Care is mostly supportive and includes supplemental oxygen, treatment of fungal and bacterial coinfections and reduction of immune suppression. Neither IVIG nor inhaled ribavirin have shown to reduce duration of PIV shedding or PIVrelated mortality in SCT recipients [5]. In a recent study, Seo et al. described ribavirin use to be associated with lowering of overall mortality [16]. However, this study failed to show any benefit in deaths due to respiratory failure or in patients with BAL confirmed LRTI. Other studies have consistently failed to show efficacy of ribavirin in HSCT recipients $[1,5,15]$.

DAS181 (Ansun BioPharma, Inc., San Diego, California) is a novel sialidase fusion protein that has shown in vitro efficacy against PIV in a cotton rat infection model as well as in lung transplant and HSCT recipients [8, 10-14]. Compared with previously published cases of use of DAS181 for PIV3 in adult HSCT recipients (Table 1), Case 1 presented very early after transplant and failed to engraft despite growth factor stimulation which played a significant role in her early demise despite receipt of a 5-day course of DAS181.

While optimal treatment duration is unknown, it is possible that a longer treatment duration prevented recurrence of infection in Case 2. In previously reported cases in HSCT recipients, a 5-day course of nebulized DAS181 was successful in clearing the infection in two patients with severe PIV LRTI requiring mechanical ventilation [10]. However, one of these patients developed recurrence of PIV and died. The authors 


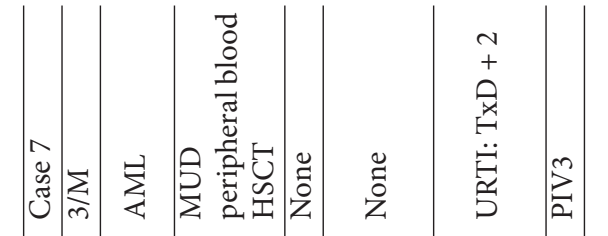

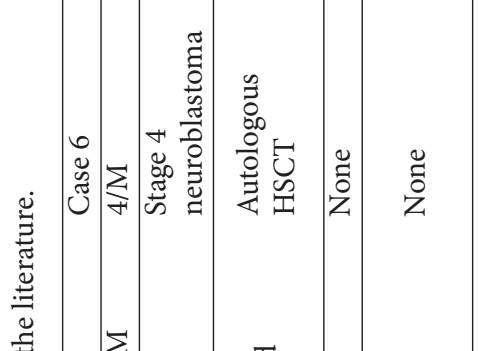

ㄴํㄴ

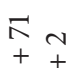

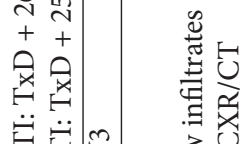

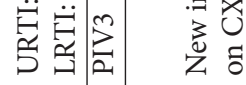

$\stackrel{+}{+}$

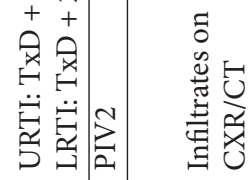

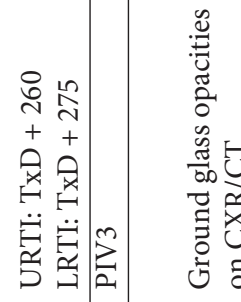

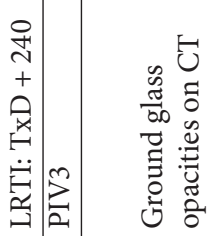

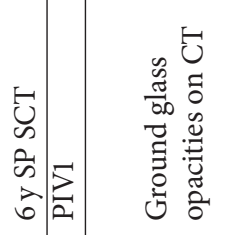

急

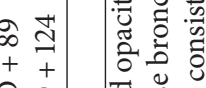

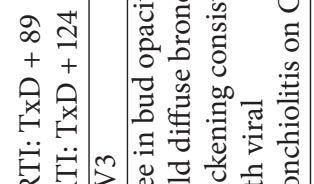

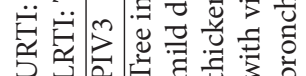

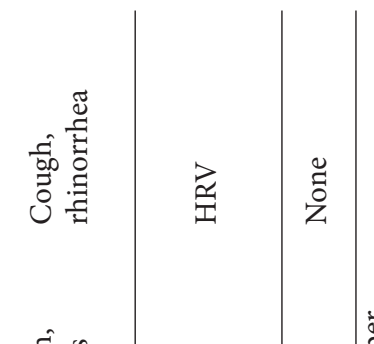

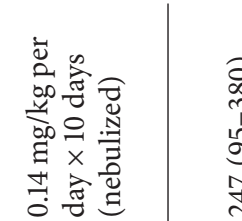

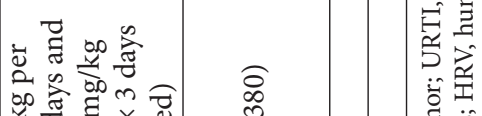

ठำ

㤹

记

(a)

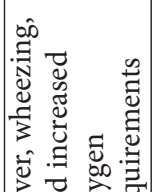

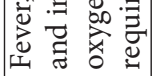

善

竞

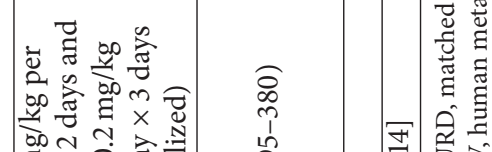

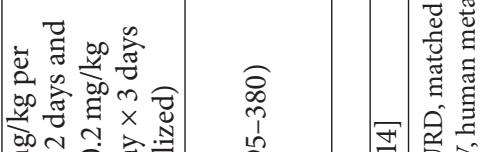

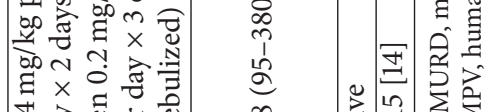


suggested that a longer treatment course might reduce risk of relapse of PIV in these critically ill patients $[10,11]$.

The adverse effects of DAS181 treatment were mild and self-limited in our patients and did not impact duration of treatment. Transient elevations in alkaline phosphatase were seen in both cases as described in the literature.

Limitations of our study include description of only two critically ill allogeneic HSCT recipients who received DAS181. Our data would have been strengthened with serial viral load studies. In the absence of this information, we are unable to unequivocally attribute the role of DAS181 in viral clearance.

\section{Conflict of Interests}

One of the authors R. B. Moss is an employee of Ansun Biopharma, the company developing DAS181. The rest of the authors have nothing to disclose.

\section{References}

[1] M. Boeckh, "The challenge of respiratory virus infections in hematopoietic cell transplant recipients," British Journal of Haematology, vol. 143, no. 4, pp. 455-467, 2008.

[2] C. B. Hall, "Respiratory syncytial virus and parainfluenza virus," The New England Journal of Medicine, vol. 344, no. 25, pp. 19171928, 2001.

[3] C. Renaud and A. P. Campbell, "Changing epidemiology of respiratory viral infections in hematopoietic cell transplant recipients and solid organ transplant recipients," Current Opinion in Infectious Diseases, vol. 24, no. 4, pp. 333-343, 2011.

[4] J. Englund, T. Feuchtinger, and P. Ljungman, "Viral infections in immunocompromised patients," Biology of Blood and Marrow Transplantation, vol. 17, no. 1, supplement, pp. S2-S5, 2011.

[5] W. Garrett Nichols, L. Corey, T. Gooley, C. Davis, and M. Boeckh, "Parainfluenza virus infections after hematopoietic stem cell transplantation: risk factors, response to antiviral therapy, and effect on transplant outcome," Blood, vol. 98, no. 3, pp. 573-578, 2001.

[6] M. P. Malakhov, L. M. Aschenbrenner, D. F. Smee et al., "Sialidase fusion protein as a novel broad-spectrum inhibitor of influenza virus infection," Antimicrobial Agents and Chemotherapy, vol. 50, no. 4, pp. 1470-1479, 2006.

[7] R. B. Moss, C. Hansen, R. L. Sanders, S. Hawley, T. Li, and R. T. Steigbigel, "A phase II study of DAS181, a novel host directed antiviral for the treatment of influenza infection," The Journal of Infectious Diseases, vol. 206, no. 12, pp. 1844-1851, 2012.

[8] B. G. Jones, R. T. Hayden, and J. L. Hurwitz, "Inhibition of primary clinical isolates of human parainfluenza virus by DAS181 in cell culture and in a cotton rat model," Antiviral Research, vol. 100, no. 2, pp. 562-566, 2013.

[9] A. Moscona, M. Porotto, S. Palmer et al., "A recombinant sialidase fusion protein effectively inhibits human parainfluenza viral infection in vitro and in vivo," The Journal of Infectious Diseases, vol. 202, no. 2, pp. 234-241, 2010.

[10] S. Chalkias, M. R. Mackenzie, C. Gay et al., "DAS181 treatment of hematopoietic stem cell transplant patients with parainfluenza virus lung disease requiring mechanical ventilation," Transplant Infectious Disease, vol. 16, no. 1, pp. 141-144, 2014.
[11] Y.-B. Chen, J. P. Driscoll, S. L. McAfee et al., "Treatment of parainfluenza 3 infection with DAS181 in a patient after allogeneic stem cell transplantation," Clinical Infectious Diseases, vol. 53, no. 7, pp. e77-e80, 2011.

[12] D. R. Drozd, A. P. Limaye, R. B. Moss et al., "DAS181 treatment of severe parainfluenza type 3 pneumonia in a lung transplant recipient," Transplant Infectious Disease, vol. 15, no. 1, pp. E28E32, 2013.

[13] B. B. Guzmán-Suarez, M. W. Buckley, E. T. Gilmore et al., "Clinical potential of DAS181 for treatment of parainfluenza3 infections in transplant recipients," Transplant Infectious Disease, vol. 14, no. 4, pp. 427-433, 2012.

[14] A. Waghmare, T. Wagner, R. Andrews et al., "Successful treatment of parainfluenza virus respiratory tract infection with DAS181 in 4 immunocompromised children," Journal of the Pediatric Infectious Diseases Society, vol. 4, no. 2, pp. 114-118, 2015.

[15] R. F. Chemaly, S. S. Hanmod, D. B. Rathod et al., "The characteristics and outcomes of parainfluenza virus infections in 200 patients with leukemia or recipients of hematopoietic stem cell transplantation," Blood, vol. 119, no. 12, pp. 2738-2745, 2012.

[16] S. Seo, H. Xie, A. P. Campbell et al., "Parainfluenza virus lower respiratory tract disease after hematopoietic cell transplant: viral detection in the lung predicts outcome," Clinical Infectious Diseases, vol. 58, no. 10, pp. 1357-1368, 2014. 


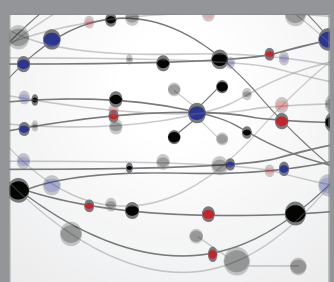

The Scientific World Journal
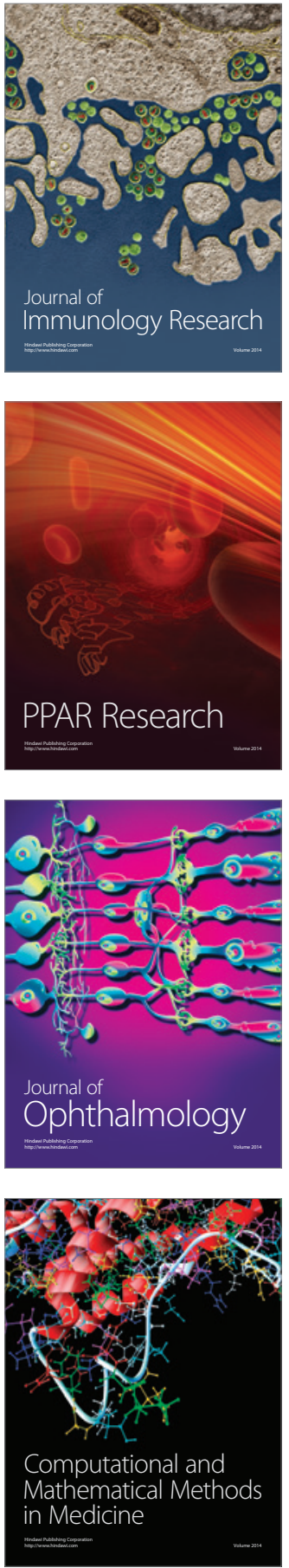

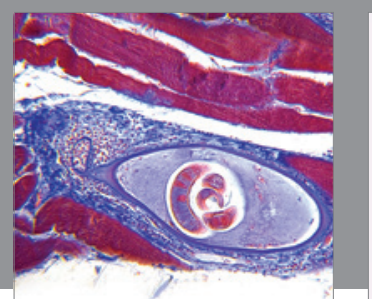

Gastroenterology Research and Practice

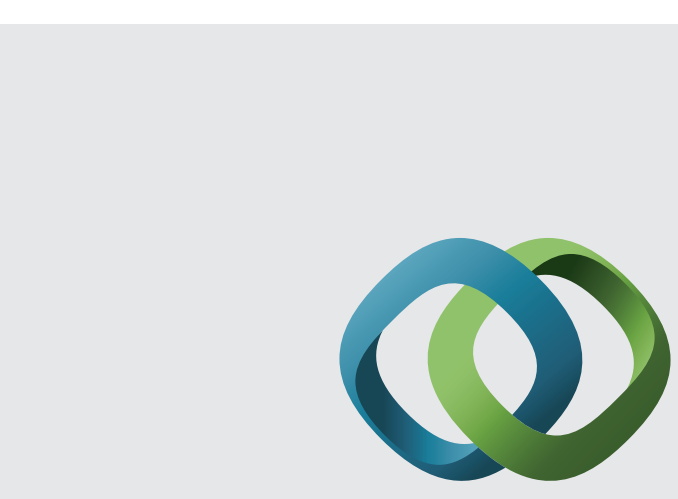

\section{Hindawi}

Submit your manuscripts at

http://www.hindawi.com
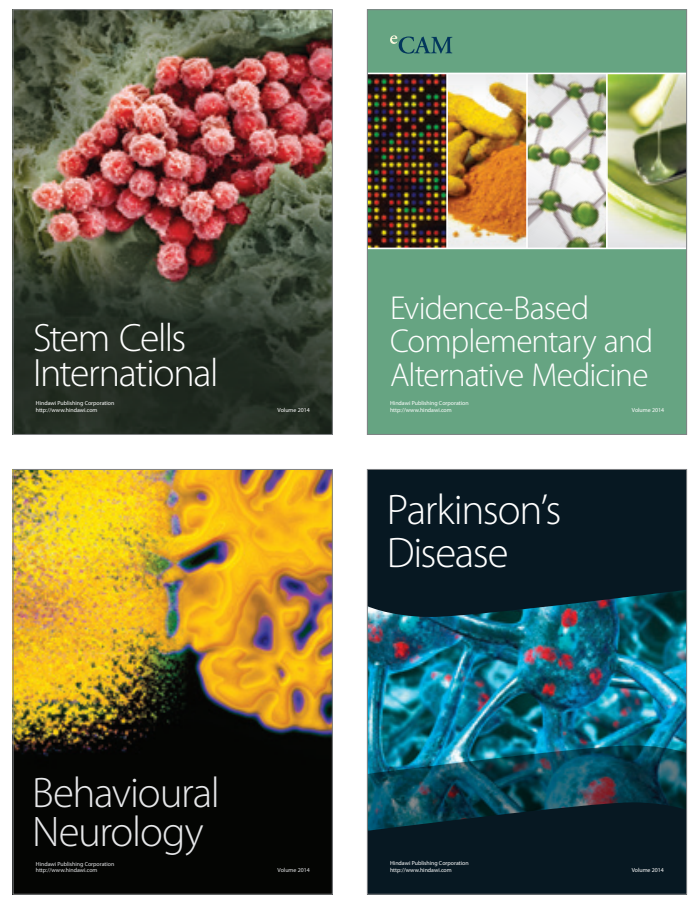
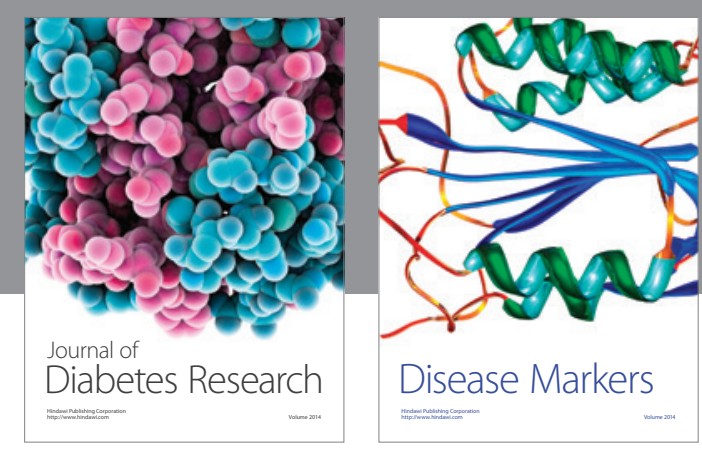

Disease Markers
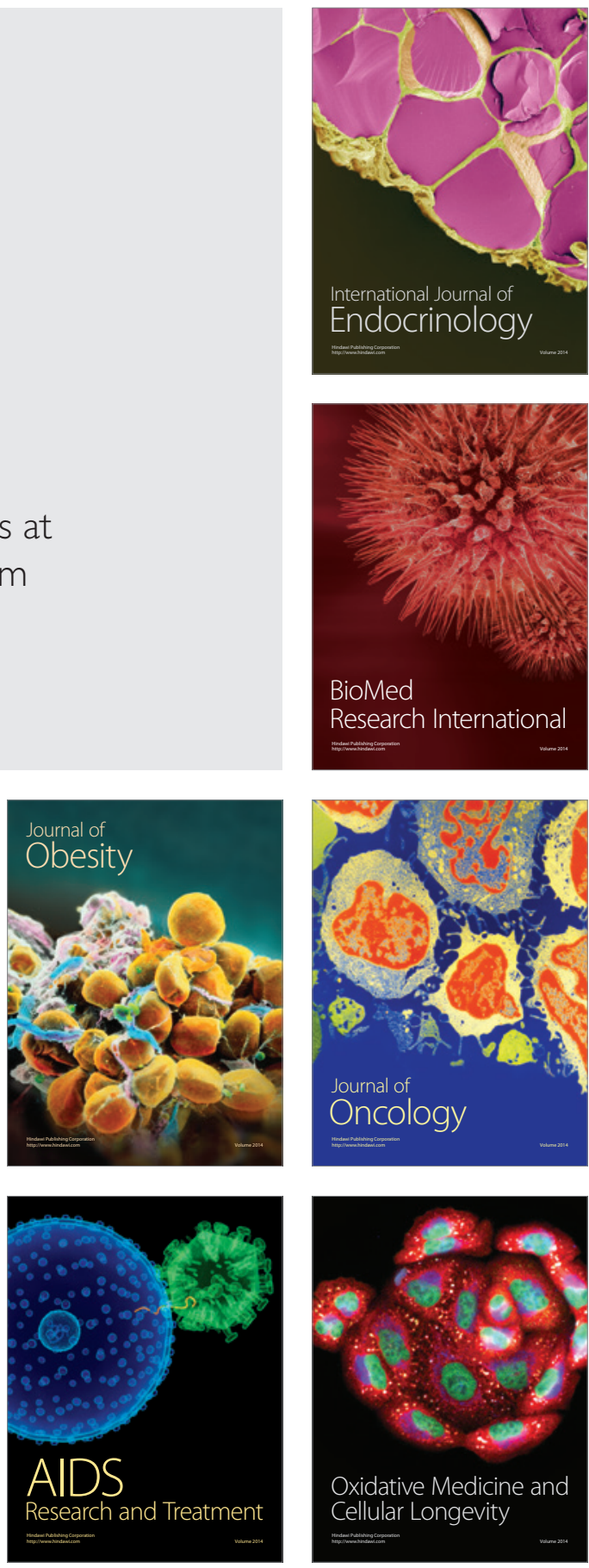